Research Paper

\title{
Knockout of the Histone Demethylase Kdm3b Decreases Spermatogenesis and Impairs Male Sexual Behaviors
}

\author{
Zhaoliang Liu'1,2, Mario G. Oyola ${ }^{1}$, Suoling Zhou ${ }^{1}$, Xian Chen ${ }^{1}$, Lan Liao ${ }^{1}$, Jean Ching-Yi Tien ${ }^{1}$, Shailaja K. \\ $\mathrm{Mani}^{1}$, Jianming $\mathrm{X} \mathrm{u}^{1,3} \bowtie$ \\ 1. Department of Molecular and Cellular Biology, Baylor College of Medicine, Houston, TX, USA. \\ 2. Institute of Cancer Research, Harbin Medical University, Harbin, China. \\ 3. Institute for Cancer Medicine and College of Basic Medical Sciences, Sichuan Medical University, Luzhou, Sichuan, China \\ $\triangle$ Corresponding author: Jianming Xu, PhD, Department of Molecular and Cellular Biology, Baylor College of Medicine, One Baylor Plaza, Houston, \\ TX 77030. Phone: 713-798-6199; Fax: 713-798-3017; E-mail: jxu@bcm.edu
}

( 2015 Ivyspring International Publisher. Reproduction is permitted for personal, noncommercial use, provided that the article is in whole, unmodified, and properly cited. See http://ivyspring.com/terms for terms and conditions.

Received: 2015.09.08; Accepted: 2015.10.04; Published: 2015.11.25

\begin{abstract}
$\mathrm{Kdm} 3 \mathrm{~b}$ is a JmjC domain-containing histone $\mathrm{H} 3(\mathrm{H} 3)$ demethylase and its physiological functions are largely unknown. In this study, we found that $\mathrm{Kdm} 3 \mathrm{~b}$ protein is highly expressed in multiple cell types in the mouse testes, including Leydig cells, Sertoli cells, spermatogonia and spermatocytes at different differentiation stages. We also observed $\mathrm{Kdm} 3 \mathrm{~b}$ protein in the epithelial cells of the caput epididymis, prostate and seminal vesicle. Breeding tests revealed that the number of pups produced by the breeding pairs with $K d m 3 b$ knockout ( $K d m 3 b K O$ ) males and wild type (WT) females was reduced $68 \%$ because of the decreased number of litters when compared with the breeding pairs with WT males and females. Further analysis demonstrated that $\mathrm{Kdm} 3 \mathrm{bKO}$ male mice produced $44 \%$ fewer number of mature sperm in their cauda epididymides, displaying significantly reduced sperm motility. No significant differences in the circulating concentration of testosterone and the expression levels of androgen receptor and its representative target genes in the testis were observed. However, the circulating levels of $17 \beta$-estradiol, a modulator of sperm maturation and male sexual behaviors, was markedly reduced in Kdm3bKO male mice. Strikingly, abrogation of $K d m 3 b$ in male mice significantly increased the latencies to mount, intromit and ejaculate and decreased the number of mounts and intromissions, largely due to their loss of interest in female odors. These findings indicate that $\mathrm{Kdm} 3 \mathrm{~b}$ is required for normal spermatogenesis and sexual behaviors in male mice.
\end{abstract}

Key words: Kdm3b, knockout mice, testis, reproductive function, sexual behavior

\section{Introduction}

The male reproductive system consists of multiple organs, including testis, epididymis, prostate and seminal vesicle. In the testis, Leydig cells located outside of the seminiferous tubules produce testosterone, the essential steroid hormone required for spermatogenesis and male sexual behaviors. Sertoli cells anchored on the inner face of the seminiferous basement membrane support all germ cells in the seminiferous epithelium and nourish the proliferation and differentiation of these germ cells [1]. During spermato- genesis in mouse, the preleptotene germ cells derived from spermatogonia proliferation and differentiation undergo the first meiosis step and sequentially develop into leptotene, zygotene, pachytene and diplotene germ cells as well as the secondary spermatocytes. The haploid spermatids formed following the rapid second meiosis step eventually develop into spermatozoa with a condensed nucleus, an acrosome, and a flagellum via the round and elongating spermatid phases [1]. The spermatozoa travel to the lumen 
of cauda epididymis for motility acquisition and storage. Sperm development and maturation require steroid hormones, especially testosterone and estrogen. It has been shown that disruption of the androgen receptor (AR) abolishes spermatogenesis and knockout of either the aromatase or the estrogen receptor inhibits spermatogenesis and spermatozoa maturation $[2,3]$.

Recent studies revealed that epigenetic regulation is also a key mechanism governing spermatogenesis male germ cell development [4-6]. Epigenetic regulation can be implicated in changes of the histone and DNA modification enzyme activities and/or alterations of the epigenetic codes and readers of both common histones and testis-specific histone variants, all of which can be important for spermatogenesis [7]. Among all the epigenetic histone codes, the methylation status of the histone $\mathrm{H} 3$ lysine 9 (H3K9) appears to be one of the most important epigenetic modifications in the testis. We have shown that the mono-, diand tri-methylation levels of $\mathrm{H} 3 \mathrm{~K} 9$ (H3K9me1/2/3) in the germ cells undergo dynamic changes during spermatogenesis [4]. Each one of the several histone methyltransferases and demethylases including Suv39h1/2, G9a, ESET, Kdm3a, and Jmjd1c can modify the methylation of $\mathrm{H} 3 \mathrm{~K} 9$ and appears to be indispensable during spermatogenesis [4, 8-11]. However, the expression pattern and role of $\mathrm{Kdm} 3 \mathrm{~b}$, a close family member of Kdm3a and Jmjd1c [12], in the testis and spermatogenesis have not been studied.

$\mathrm{Kdm} 3 \mathrm{~b}$ is a histone $\mathrm{H} 3$ demethylase that specifically catalyzes the demethylation of $\mathrm{H} 3 \mathrm{~K} 9 \mathrm{me} 1 / 2$ [12]. Several studies have associated the role of $\mathrm{Kdm} 3 \mathrm{~b}$ with cell growth and transformation. Specifically, $\mathrm{Kdm} 3 \mathrm{~b}$ plays a potential tumor suppressor role in myeloid leukemia, myelodysplasia and breast cancer, but it may promote acute promyelocytic leukemia [12-15]. We have recently generated $K d m 3 b$ knockout (Kdm3bKO) mice. We found that knockout of $K d m b 3$ restricted the postnatal somatic growth of these mice. At the molecular level, $\mathrm{Kdm} 3 \mathrm{bKO}$ mice exhibited decreased levels of the insulin growth factor binding protein-3 (IGFBP-3) expression, resulting in a significantly decreased IGF-1 stability in the blood circulation and a restriction of postnatal somatic growth [16]. We also found that $\mathrm{Kdm} 3 \mathrm{bKO}$ female mice were infertile because of irregular estrous cycles and decreased ovulation, fertilization, and uterine decidual response [16].

In this study, we defined the expression patterns of the $\mathrm{Kdm} 3 \mathrm{~b}$ protein in the male reproductive organs and the physiological functions of Kdm3b in the male reproductive system through characterizing the reproductive phenotypes of the male $\mathrm{Kdm} 3 \mathrm{bKO}$ mice. Our results demonstrate that $\mathrm{Kdm} 3 \mathrm{~b}$ is required for normal spermatogenesis and male sexual behaviors.

\section{Materials and Methods}

\section{Mice}

The wild type (WT) control mice and the heterozygous and homozygous Kdm3b knockout (Kdm3bKO) mice were produced from the heterozygous breeding pairs as we described previously [16]. All mice used in this study had a mixed C57BL/6J and $129 \mathrm{SvEv} / \mathrm{j}$ strain background. All mice were housed in our facility with a 12 hours light and 12 hours dark cycle and allowed free access to water and food. Animals used in the reproductive behavior studies were housed under a reverse light cycle (12 hours dark/12 hours light) with lights off at 6:00 am. Behavior assessments were performed between 9:00 am and 3:00 $\mathrm{pm}$. All the animal protocols were approved by the Institutional Animal Care and Use Committee of Baylor College of Medicine and were conducted in accordance with the National Institutes of Health Guidelines.

\section{Histological analysis and immunohistochem- istry (IHC)}

The testes, epididymides, prostates and seminal vesicles were isolated from sacrificed WT and $\mathrm{Kdm} 3 \mathrm{bKO}$ male mice and fixed in $4 \%$ paraformaldehyde overnight at $4{ }^{\circ} \mathrm{C}$. Paraffin sections were prepared from these tissues as described previously [16, 17]. These sections were stained with Hematoxylin and Eosin (H\&E) and then, examined and imaged under a microscope. IHC was performed as described previously [4, 18]. Briefly, the de-paraffinized and re-hydrated tissue sections were treated in the $10 \mu \mathrm{M}$ sodium citrate buffer at $95^{\circ} \mathrm{C}$ for 10 minutes for antigen retrieval. The treated tissue slides were further incubated in the methanol solution with $3 \% \mathrm{H}_{2} \mathrm{O}_{2}$ for quenching the endogenous peroxidase. After blocking with $10 \%$ goat serum and $1 \%$ bovine serum albumin (BSA), the tissue slides were incubated with the primary antibody and then the biotin-conjugated secondary antibody. The bound secondary antibody was visualized by sequentially incubating with the Avidin-conjugated horseradish peroxidase (HRP) (AK-5200, Vector Laboratories) and its substrate diaminobenzidine (SK-4100, Vector Laboratories). The tissue slides were counter-stained with hematoxylin, sealed in Permount and examined by bright field microscopy.

\section{Antibodies}

This study used antibodies against $\mathrm{Kdm} 3 \mathrm{~b}$ (2621S, Cell signaling), histone H3 (H3) (ab1791, Abcam), H3K9me1 (ab9045, Abcam), H3K9me2 (07-441, Upstate), H3K9me3 (07-442, Upstate), $\beta$-actin 
(A2228, clone ac-74, Sigma-Aldrich).

\section{Quantitative RT-PCR (QPCR)}

RNA samples were prepared from the testes of WT and Kdm3bKO mice by using the Trizol Reagent (15596-018, Invitrogen). Total RNAs were reverse-transcribed with random primers. QPCR reactions were performed in triplicate by using the gene-specific primer pairs and the matched universal mouse probe sets (Roche Applied Science, Mannheim). The relative expression levels of all genes were normalized to the level of endogenous 18S RNA.

\section{Western blot}

Protein samples were prepared from the testes of WT and Kdm3bKO mice as described previously [4, 16]. Protein concentration was measured by using the BCA Protein Assay Kit (23225, Pierce Biotechnology). Western blot analysis was carried out with $20 \mu \mathrm{g}$ protein in each sample as described previously $[4,16]$.

\section{Sperm analysis}

Sperm count and motility assay were performed as described previously [4, 19]. Briefly, one cauda epididymis was removed from each male mouse and minced in $1 \mathrm{ml}$ of phosphate buffered saline (PBS). After allowing the sperm to disperse in the solution for 5 minutes, total and motile sperms were counted with a hemacytometer under a microscope.

\section{Measurement of testosterone and 17 $\beta$-estradiol}

Blood samples were collected by periorbital puncture method from age-matched WT and $\mathrm{Kdm} 3 \mathrm{bKO}$ mice. Serum was isolated following clotting at $4^{\circ} \mathrm{C}$. The concentrations of $17 \beta$-estradiol and testosterone were measured with the ELISA kits following the manufacture's instruction (DSL-4400 and DSL-4000, Diagnostic Systems Laboratories).

\section{Male Reproductive Behavior}

Male-typical reproductive behaviors of 8-week old WT mice and heterozygous and homozygous $\mathrm{Kdm} 3 \mathrm{bKO}$ male mice were examined in the presence of sexually receptive stimulus females as described [20]. Briefly, age-matched, ovariectomized WT female mice were primed with subcutaneous injection of estradiol benzoate (EB, $0.5 \mu \mathrm{g} /$ mouse), followed by injection of progesterone $(100 \mu \mathrm{g} /$ mouse $)$ after 48 hours of EB injection. These hormone-primed mice were used as stimulus females. Male behavior tests were performed under red light during the dark phase of the reverse light cycle, 6 hours after progesterone administration. A female mouse was introduced into the home cage $(27 \times 16.5 \times 12.5 \mathrm{~cm})$ of a single-housed male. A mirror stand (mirror inclined at an angle of $45^{\circ}$ ) was used to allow for ventral viewing. Once the female was introduced, males were allowed 1800 seconds to mount, intromit or ejaculate. Once ejaculation occurred the test was terminated. If the male neither intromitted nor ejaculated within $1800 \mathrm{sec}-$ onds, this male was considered sexually inactive and given the maximum score for a latency of $1800 \mathrm{sec}$. Ejaculations were confirmed in the females by checking for vaginal plugs immediately after the testing session. Recorded measurements included the total number of mounts, intromissions, and ejaculations, as well as their latencies. The observers were blind to the treatment and genotype.

\section{Olfactory Capacity Test}

Olfactory capacity was evaluated as described previously [20]. The latency to discover a piece of Oreo ${ }^{\odot}$ cookie $\left(5 \mathrm{~mm}^{3}\right)$ hidden randomly under the fresh clean bedding material in an arena $(26 \times 15 \times 12$ $\mathrm{cm})$ was observed for $5 \mathrm{~min}$ and recorded. If the discovery time exceeded $5 \mathrm{~min}$, the test was terminated.

\section{Olfactory Preference Test}

A male mouse's olfactory preference for bedding from a male, behavioral estrous female, or clean bedding was examined as described previously [20]. The time spent by each mouse investigating each bowl containing the bedding was recorded for a total of 10 min.

\section{Results}

\section{$\mathrm{Kdm} 3 \mathrm{~b}$ is highly expressed in the male repro- ductive organs}

As an initial step to investigate the function of $\mathrm{Kdm} 3 \mathrm{~b}$ in the male reproductive function, we examined the tissue and cell type-specific expression patterns of $\mathrm{Kdm} 3 \mathrm{~b}$ in the male mouse reproductive organs. Western blot analysis detected a strong $200 \mathrm{kDa}$ $\mathrm{Kdm} 3 \mathrm{~b}$ band from the testis extract of wild type (WT) mice but not from the testis extract of Kdm3b knockout mice (Fig. 1A). Immunohistochemistry (IHC) staining with $\mathrm{Kdm} 3 \mathrm{~b}$ antibody detected $\mathrm{Kdm} 3 \mathrm{~b}$ protein expressed in Leydig cells, Sertoli cells and different types of germ cells in the testis of WT mice but not in these cells in the testis of $\mathrm{Kdm} 3 \mathrm{~b} \mathrm{KO}$ mice (Fig. 1B). IHC analysis of the seminiferous epithelium further revealed that $\mathrm{Kdm} 3 \mathrm{~b}$ protein is expressed in the spermatogonia and different stage spermatocytes including preleptotene, leptotene, zygotene, and stages I-IX pachytene spermatocytes. The strongest Kdm3b immunoreactivity was found in stage VI pachytene cells, while spermatocytes during stages X-XII showed no detectable Kdm3b (Fig. 1C). Kdm3b protein was also not detected in the round and elongating spermatids at any stages (Fig. 1C). In the epididymis, 
$\mathrm{Kdm} 3 \mathrm{~b}$ protein was detected at a high level in the caput epididymis, but its IHC signal was low in the cauda epididymis (Fig. 2A and B). In addition, low levels of $\mathrm{Kdm} 3 \mathrm{~b}$ protein were detected in the epithelial cells of the prostate and seminal vesicle (Fig. 2C and $\mathrm{D}$ ). Together, the highly expressed $\mathrm{Kdm} 3 \mathrm{~b}$ in the testis and caput epididymis suggests that $\mathrm{Kdm} 3 \mathrm{~b}$ may play a role in the male reproductive function.

\section{Kdm3b knockout male mice are subfertile}

To define the genetic function of $\mathrm{Kdm} 3 \mathrm{~b}$ in the male reproductive function, we took advantage from $\mathrm{Kdm} 3 \mathrm{bKO}$ mice generated recently in our laboratory [16] and compared the reproductive capability of male $\mathrm{Kdm} 3 \mathrm{bKO}$ mice with age-matched male wild type (WT) mice. After 5 male WT mice had been paired with 5 WT female mice at a 1:1 ratio in each cage for 4 months, a total of 148 pups (29.6 pups/breeding pair) were produced. However, the same breeding paradigm with 5 male $\mathrm{Kdm} 3 \mathrm{bKO}$ and $5 \mathrm{WT}$ female mice only produced a total of 47 pups ( 9.4 pups/breeding pair) (Table 1). Further analysis of the breeding data revealed that each breeding pair with the male $\mathrm{Kdm} 3 \mathrm{bKO}$ mouse produced only one litter in average, which was significantly less than the 3.6 litters produced in average by each breeding pair with the male WT mouse. The average number of pups per litter was not significantly different between these two breeding groups (Table 1). These results indicate that $\mathrm{Kdm} 3 \mathrm{~b}$ is required for normal male reproductive function. Disruption of $\mathrm{Kdm} 3 \mathrm{~b}$ function compromises the male reproductive capability mainly reflected by the reduced number of litters produced by the breeding pairs with the male $\mathrm{Kdm} 3 \mathrm{bKO}$ mice.
A

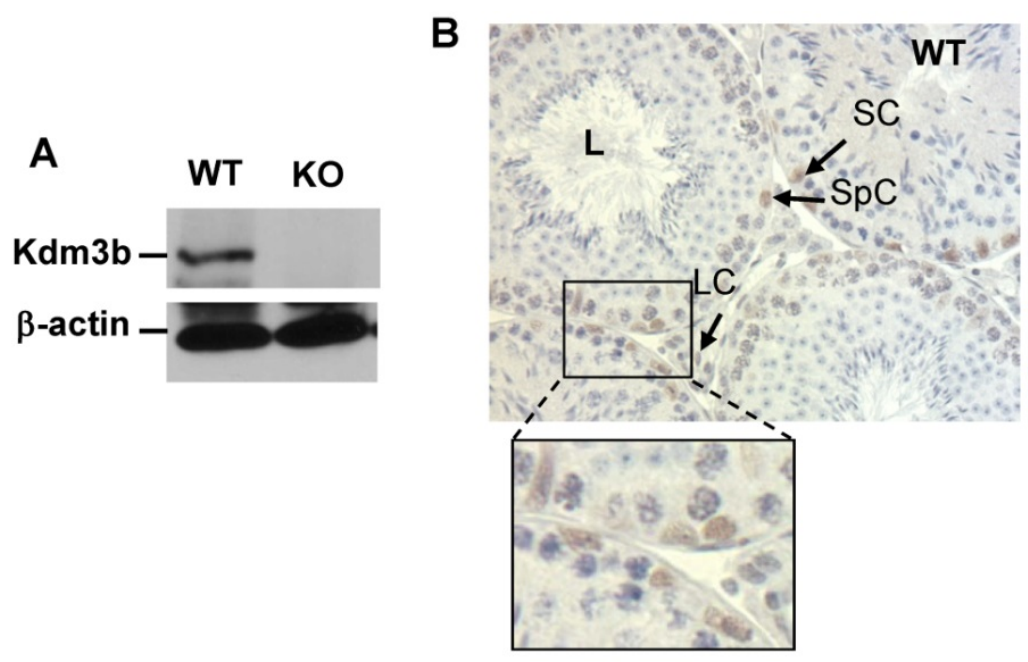

C

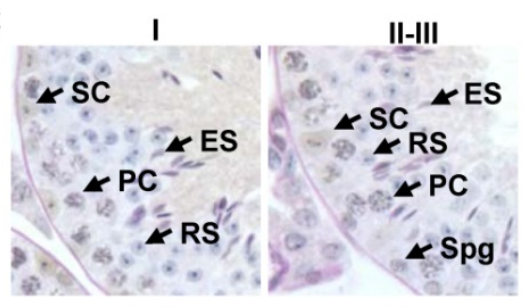

VIII

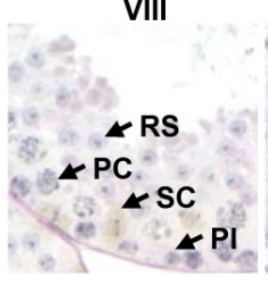

IX

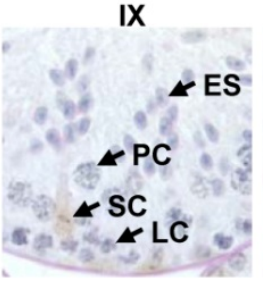

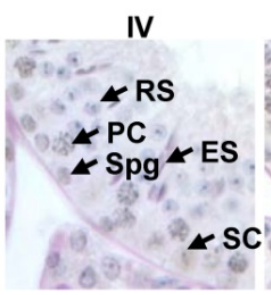

$\mathbf{X}$

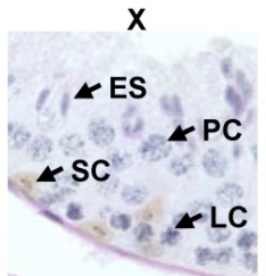

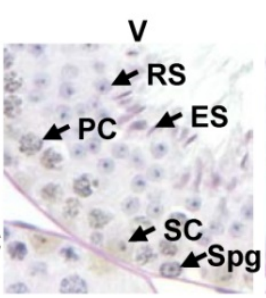
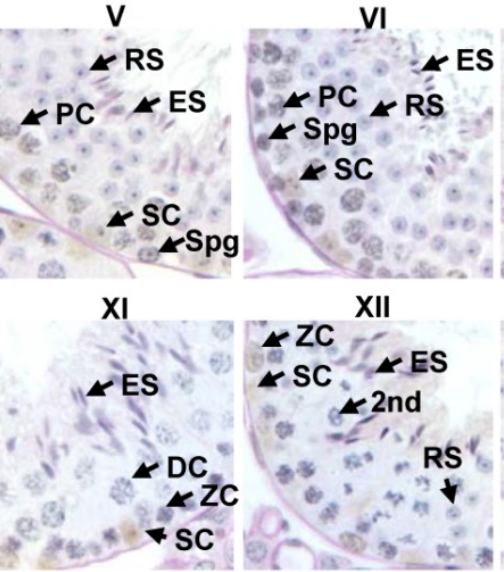

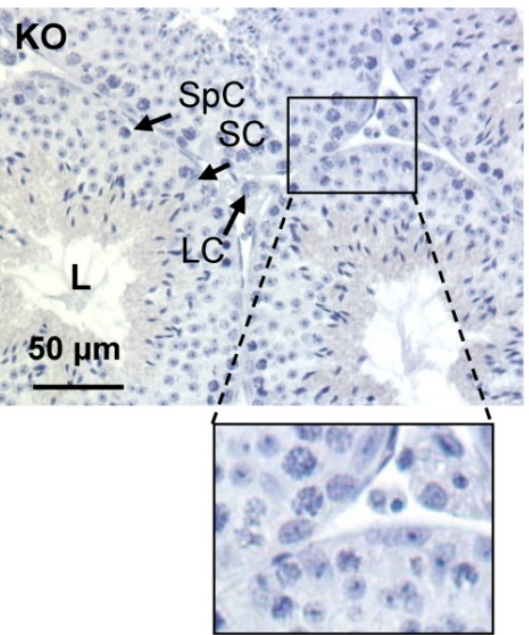

Figure 1. Analysis of Kdm3b protein expression in the mouse testis. A. Western blot analysis of Kdm $3 \mathrm{~b}$ protein. The $200 \mathrm{kD} K \mathrm{dm} 3 \mathrm{~b}$ protein was detected in the tissue lysate of WT mouse testis. The tissue lysate of Kdm3bKO $(\mathrm{KO})$ mouse testis served as a negative control. The analysis of $\beta$-actin served as a loading control. B. Detection of $\mathrm{Kdm} 3 \mathrm{~b}$ by IHC (brown) in the testis sections prepared from 12-14-week old male mice $(\mathrm{n}=3)$. The testis section prepared from age-matched $\mathrm{Kdm} 3 \mathrm{bKO}$ mice was used as a negative control for IHC. L, the lumen of the seminiferous tubule; LC, Leydig cell; SC, Sertoli cell; SpC, spermatocyte. C. Analysis of Kdm3b expression in germ cells by IHC at different spermatogenesis stages of the seminiferous tubules. Testis sections with germ cells characteristic of stages I to XII from WT mice were used for Kdm3b IHC. Stage-matched sections from Kdm3bKO mice were used as negative controls for IHC, although only the result from one-stage section was shown as indicated by "KO, V". L, LC, SC and SpC, see explanations above for Panel B; Spg, spermatogonia; Pl, preleptotene cells; ZC, zygotene cells; PC, pachytene cells; 2nd, secondary spermatocyte; RS, round spermatid; ES, elongating spermatid. 
A

WT

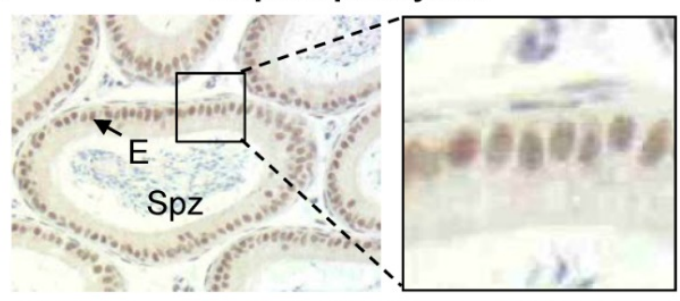

KO

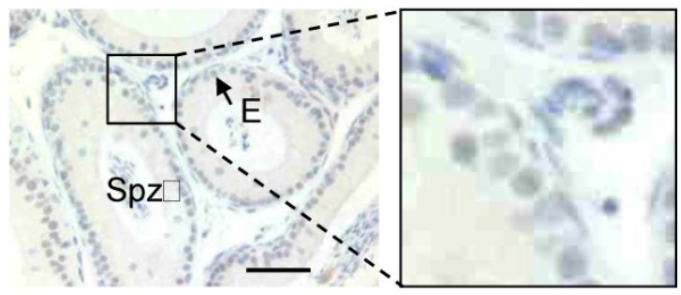

C

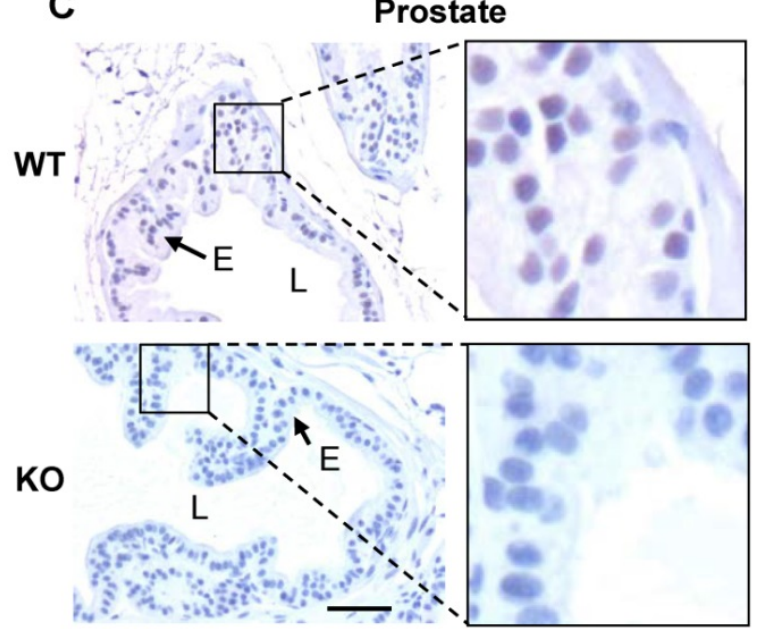

B

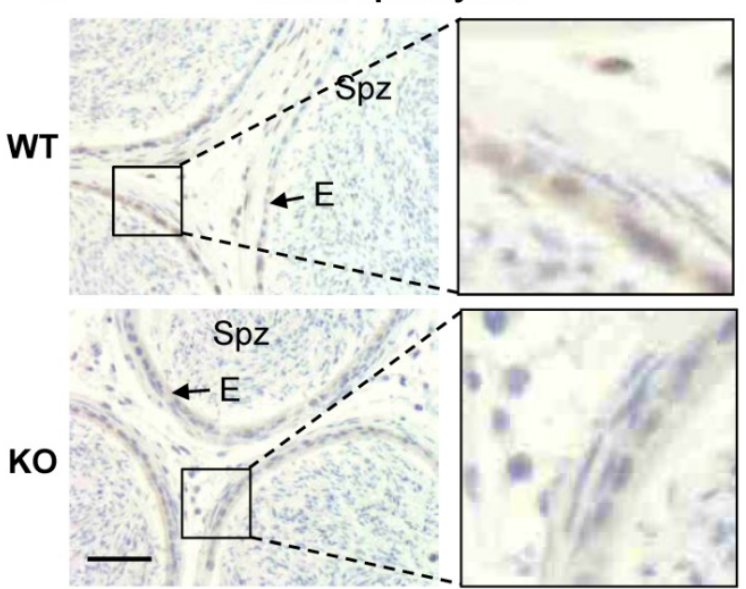

D

Seminal vesicle

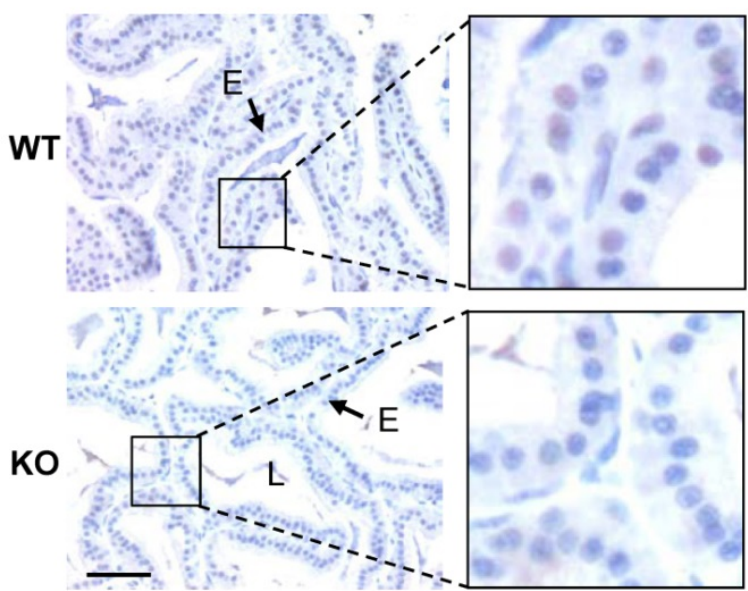

Figure 2. Immunohistochemistry analysis of Kdm3b protein expression in the epididymis, prostate and seminal vesicle. A. Detection of the high level Kdm3b protein expression by $\mathrm{IHC}$ in the epithelial cells $(\mathrm{E})$ of the caput epididymis of WT mice. The section prepared from the caput epididymis of Kdm3bKO (KO) mice served as a negative control for IHC. Spz, spermatozoa. B. Detection of the low level Kdm3b protein expression by IHC in the epithelial cells (E) of the cauda epididymis of WT mice. The section prepared from the cauda epididymis of Kdm3bKO (KO) mice served as a negative control for IHC. C and D. Detection of Kdm3b protein expression in the epithelial cells $(E)$ of the prostate and seminal vesicle in WT mice. Tissue sections prepared from the prostate and seminal vesicle of Kdm3bKO mice served as negative controls for IHC. $\mathrm{L}$, the lumen of the prostate or seminal vesicle glands. The scale bars in all panels represent $25 \mu \mathrm{m}$.

Table 1. Evaluation of the $\mathrm{Kdm} 3 \mathrm{~b}$ null mice fertility by mating experiment.

\begin{tabular}{|c|c|c|c|c|c|c|}
\hline Mating & Pairs & $\begin{array}{l}\text { Total } \\
\text { Pups }\end{array}$ & Pups/Pair & $\begin{array}{l}\text { Total } \\
\text { Litters }\end{array}$ & Litters/Pair & Pups/Litter \\
\hline$\sigma^{2} \mathrm{WT} \times$ 우 WT & 5 & 148 & $29.6 \pm 9.4$ & 18 & $3.6 \pm 1.1$ & $8.2 \pm 2.6$ \\
\hline${ }^{7} \mathrm{KO} \times$ 우 $\mathrm{WT}$ & 5 & $47^{* *}$ & $9.4 \pm 8.9^{*}$ & $5^{\star *}$ & $1.0 \pm 1.0^{*}$ & $9.4 \pm 2.4$ \\
\hline
\end{tabular}

The breeding pairs with the indicated genotypes were housed together for 4 months and the reproductive data of each breeding pair were recorded. * $\mathrm{p}<0.05$; ${ }^{* *}, \mathrm{p}<0.01$

\section{Kdm3b KO mice exhibit decreased sperm number and motility}

To search for the causes responsible for the subfertile phenotype of the male $\mathrm{Kdm} 3 \mathrm{bKO}$ mice, we first compared their testis weight, sperm number and sperm motility with those of age-matched male WT mice. Although the average weight of testes in $\mathrm{Kdm} 3 \mathrm{~b}$ knockout mice was lesser than that in age-matched WT mice, the ratios of testis weight to body weight were the same for these two groups of male mice (Fig. $3 \mathrm{~A})$, indicating that the lesser net weight of testes in $\mathrm{Kdm} 3 \mathrm{~b}$ knockout mice is attributed to their smaller body sizes [16]. Next, we compared the histology between the testes of age-matched WT and Kdm3b knockout mice by H\&E staining. We found that all types of germ cells at all developmental stages were present in the testes of $\mathrm{Kdm} 3 \mathrm{~b}$ knockout mice, and the morphology and cellular components of their seminiferous epithelium were similar to those of the age-matched WT mice (Fig. 3B and data not shown). Furthermore, we also counted the numbers of total and motile sperm isolated from the caudal epididymides of age-matched WT and Kdm3b knockout mice. We found that the average number of sperm in each WT epididymis was about 17.8 million, while the 
average number of sperm in each $\mathrm{Kdm} 3 \mathrm{~b}$ knockout epididymis was only about 9.96 millions, which was reduced $44 \%$ versus that in WT mice (Fig. 3C). The percentage of motile sperm isolated from the epididymides of $\mathrm{Kdm} 3 \mathrm{~b}$ knockout mice was also significantly reduced when compared with the percentage of motile sperm isolated from the epididymides of age-matched WT mice (Fig. 3D). These results suggest that $\mathrm{Kdm} 3 \mathrm{~b}$ deficiency partially decreases the total number of sperm and the percentage of motile sperm in mice.

\section{Kdm3b deficiency does not significantly alter the global levels of H3K9 methylation in the testis}

Since $\mathrm{Kdm} 3 \mathrm{~b}$ is a histone demethylase specific to H3K9me2 and H3K9me1 [12], we assessed whether

A

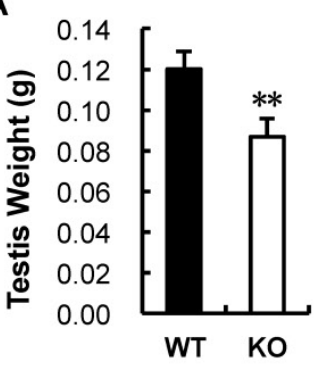

B

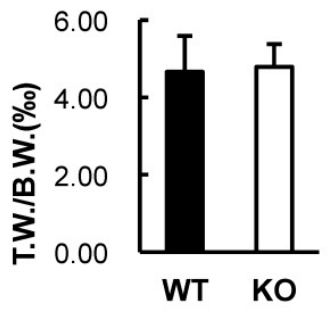

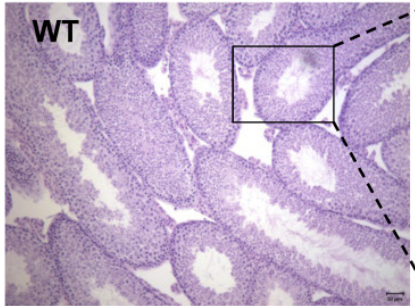

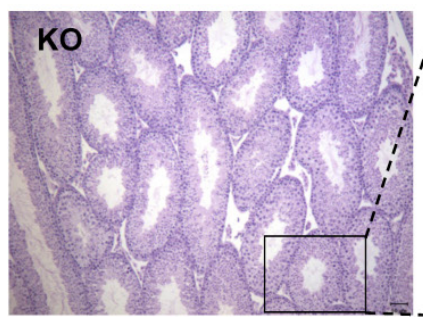

knockout of $K d m 3 b$ could change the global levels of H3K9 methylation in the testis. Western blot analysis of the testis tissue lysates did not detect obvious changes in the levels of H3K9me1, H3K9me2 and H3K9me3 (Fig. 4A). We further performed IHC to examine $\mathrm{H} 3 \mathrm{~K} 9$ methylation status in individual cells. We found that the levels of H3K9me1, H3K9me2 and $\mathrm{H} 3 \mathrm{~K} 9 \mathrm{me} 3$ were relatively high in the spermatogonia and early pachytene spermatocytes and then, gradually decreased in the further developed spermatocytes in both $\mathrm{WT}$ and $\mathrm{Kdm} 3 \mathrm{bKO}$ testes (Fig. 4B, and data not shown). These results demonstrate that knockout of $K d m 3 b$ does not cause significant changes in the global levels of $\mathrm{H} 3 \mathrm{~K} 9 \mathrm{me} 1, \mathrm{H} 3 \mathrm{~K} 9 \mathrm{me} 2$ and $\mathrm{H} 3 \mathrm{~K} 9 \mathrm{me} 3$ in the mouse testes and suggest that $\mathrm{Kdm} 3 \mathrm{~b}$ may function at specific chromatin sites or regions.

\section{C}
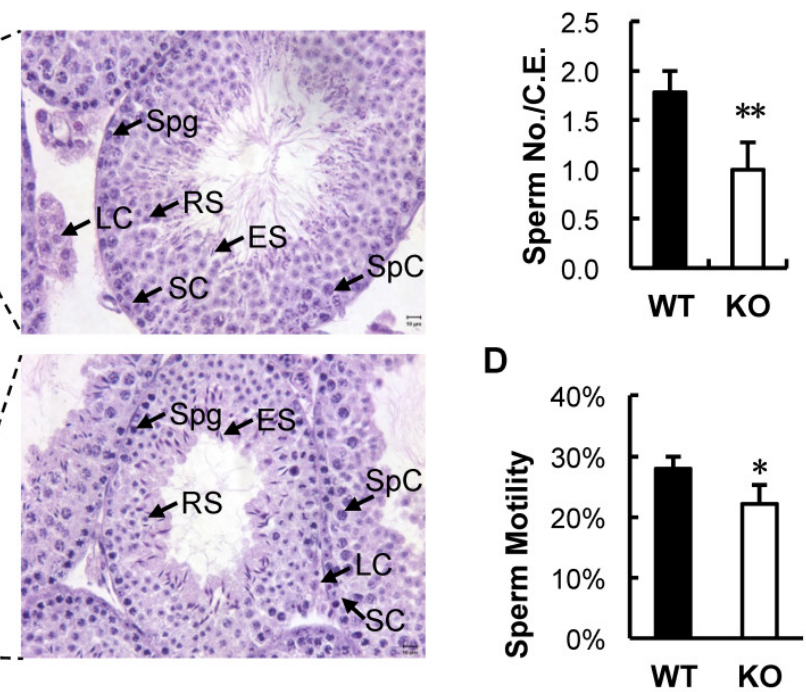

Figure 3. Analysis of the testis weight and spermatogenesis in Kdm3bKO mice. A. The average wet testis weights of 10 -week-old WT ( $\mathrm{n}=4$ ) and Kdm3bKO (KO, $n=4)$ mice (upper panel), and the average relative testis weights (T.W.) of these mice after normalized to their body weights (B.W.) (lower panel). **, $p<0.01$ by Student's $t$ test. B. Histological analysis of age-matched WT and Kdm3bKO (KO) mouse testes. Images in the left panels are shown at low magnification, while the images in the right panels are the magnified areas indicated in the left panels. LC, Leydig cell; SC, Sertoli cell; Spg, spermatogonia; SpC, spermatocyte; RS, round spermatid; ES, elongating spermatid. C. Comparison of average sperm numbers per cauda epididymis (C.E.) in 10-week-old WT $(n=4)$ and Kdm3bKO (KO) mice $(n=4)$. **, $p<0.01$ by Student's $t$ test. D. The average percentages of motile sperm in the cauda epididymides of 10 -week-old WT $(n=4)$ and Kdm3bKO $(K O)$ mice $(n=4)$. *, $p<0.05$ by Student's t test.

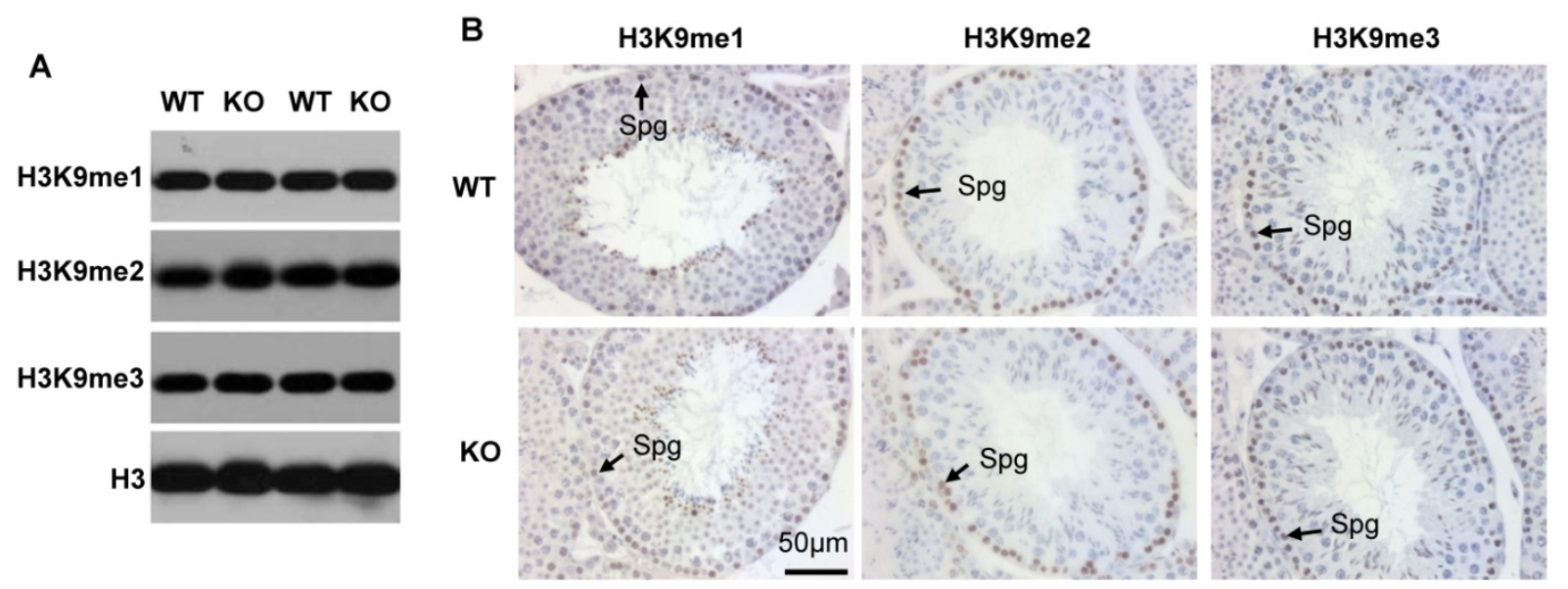

Figure 4. Analysis of $\mathbf{H 3 K} 9 \mathrm{me} \mathbf{1} / \mathbf{2} / 3$ levels in the testes of 10-week-old WT and Kdm3bKO (KO) mice. A. Western blot analysis of H3K9me1/2/3 levels in the testes of WT and $\mathrm{KO}$ mice $(n=2)$. Total $\mathrm{H} 3$ served as an internal loading control. B. Analysis of H3K 9 mel/2/3 levels by IHC. Tissue sections prepared from the testes of WT and Kdm3bKO mice $(n=3)$ were used for IHC analysis. Representative data are presented. Spg, spermatogonia. 


\section{The $17 \beta$-estradiol is decreased in the blood circulation of the male Kd3bKO mice}

Reproductive steroid hormones and their signaling pathways are essential for normal reproductive function. We first measured the serum concentrations of testosterone and found that the average testosterone level in the serum samples of the male $\mathrm{Kdm} 3 \mathrm{bKO}$ mice was much higher than that of the age-matched male WT mice (Fig. 5A). However, owing to the broad variations among individual animals within either genotype group, the difference in testosterone concentration between $\mathrm{Kdm} 3 \mathrm{bKO}$ and WT mice did not reach statistical significance. We also examined the expression levels of AR and two of its known target genes, Eppin and RhoX5 [21]. We find that these genes were expressed at similar levels in the testes of WT and Kdmb3KO mice (Fig. 5B). These results suggest that the androgen signaling pathway is functional in the male $\mathrm{Kdm} 3 \mathrm{bKO}$ mice although their testosterone levels are likely increased.

We next measured the serum concentrations of $17 \beta$-estradiol, a steroid hormone derived mainly from testosterone via aromatization and required for both male and female reproductive functions [22, 23]. We found that $17 \beta$-estradiol concentration was markedly reduced by $55 \%$ in the serum samples of the male $\mathrm{Kdm} 3 \mathrm{bKO}$ mice versus the male WT mice (Fig. 5C). These results demonstrate that $\mathrm{Kdm} 3 \mathrm{~b}$ is required for maintaining normal circulating levels of $17 \beta$-estradiol.

To understand the mechanisms responsible for the decreased $17 \beta$-estradiol in the male $\mathrm{Kdm} 3 \mathrm{bKO}$ mice, we further examined the expression levels of genes regulating or influencing estrogen synthesis including the aromatase (Cyp19A1), several hydroxysteroid dehydrogenases (Hsd17b1, Hsd17b4 and $H s d 17 b 7)[24,25]$, and the steroid 5 alpha reductase 1 (Srd5a1) [26]. However, the mRNA expression levels of these genes were not significantly altered in the testes of $\mathrm{Kdm} 3 \mathrm{bKO}$ mice when compared with those in the testes of WT mice (Fig. 5D). We also examined the expression level of the estrogen sulfotransferase (Sult1e1) that catalyzes estradiol degradation through sulfoconjugation [24]. We found that the mRNA expression level of this enzyme was comparable in the testes of the male WT and Kdm3bKO mice (Fig. 5D). In summary, Kdm3b deficiency does not affect the expression of these genes involved in estrogen synthesis. Therefore, the mechanisms responsible for the decreased estradiol in the male $\mathrm{Kdm} 3 \mathrm{bKO}$ mice remain unknown at the current time.

\section{Knockout of $K d m 3 b$ impaired male sexual be- havior}

Since the subfertile phenotype of the male $\mathrm{Kdm} 3 \mathrm{bKO}$ mice is associated with the decreased number of litters but not the number of pups per litter, we examined whether the male-specific reproductive behaviors were impaired in these mice. The latency to mount a stimulus female was significantly prolonged (1200 seconds) in Kdm3bKO mice compared to their male WT littermates mice (240 seconds). The male heterozygous $\mathrm{Kdm} 3 \mathrm{~b}$ mice also showed a slightly delayed latency (415 seconds) to mount the stimulus female (Fig. 6A). During the testing period, the number of mounts by the WT and heterozygous males were significantly higher than those of the $\mathrm{Kdm} 3 \mathrm{bKO}$ mice (Fig. 6B). Furthermore, the male $\mathrm{Kdm} 3 \mathrm{bKO}$ mice also took significantly longer time to achieve their first successful intromission compared with the male WT and heterozygous mice (Fig. 6C). Interestingly, $\mathrm{Kdm} 3 \mathrm{bKO}$ males only mounted the females 4 times and had only 1 time intromission on average (Fig. 6B and D). Moreover, in the presence of stimulus females, the male WT and heterozygous mice carried out successful ejaculation within about 820 and 515 seconds on average, while the male $\mathrm{Kdm} 3 \mathrm{bKO}$ mice showed no successful ejaculation within the entire testing period (Fig. 6E). These results demonstrate that knockout of $\mathrm{Kdm} 3 \mathrm{~b}$ severely impaired the consummatory components of the male sexual behaviors.

To examine whether the impaired reproductive behavior in the males was due to a defect in detection of olfactory pheromonal cues, we compared the odor recognition ability of the male $\mathrm{Kdm} 3 \mathrm{bKO}$ mice to that of the age-matched male WT mice. In this test, the mice were exposed to clean, soiled male or soiled behavioral estrus female bedding, and their preference was observed. Although the $\mathrm{Kdm} 3 \mathrm{bKO}$ male mice spent a little longer time than the WT and heterozygous male mice did on investigating the clean or male-soiled bedding, there were no significant differences between any two groups according to statistical analysis (Fig. 7A and B). Interestingly, while the male WT and heterozygous mice spent a longer time (about 175 seconds) on investigating female bedding, the male $\mathrm{Kdm} 3 \mathrm{bKO}$ mice only spent about 47 seconds on investigating the same bedding (Fig. 7C). To exclude the possibility that the $\mathrm{Kdm} 3 \mathrm{bKO}$ mice could be defective in their general odor recognition, we examined the olfactory capacity of the mice to find a hidden palatable object. The latency to identify the hidden palatable object (Oreo cookie) was not significantly different between any two groups of the WT, heterozygous and $\mathrm{Kdm} 3 \mathrm{bKO}$ mice (Fig. 7D). Thus, the different interest level of $\mathrm{Kdm} 3 \mathrm{bKO}$ mice to the female scent was not caused by a loss of general olfactory function. These results demonstrate that $\mathrm{Kdm} 3 \mathrm{~b}$ is also required for maintaining the motivational components of the male sexual behaviors. 

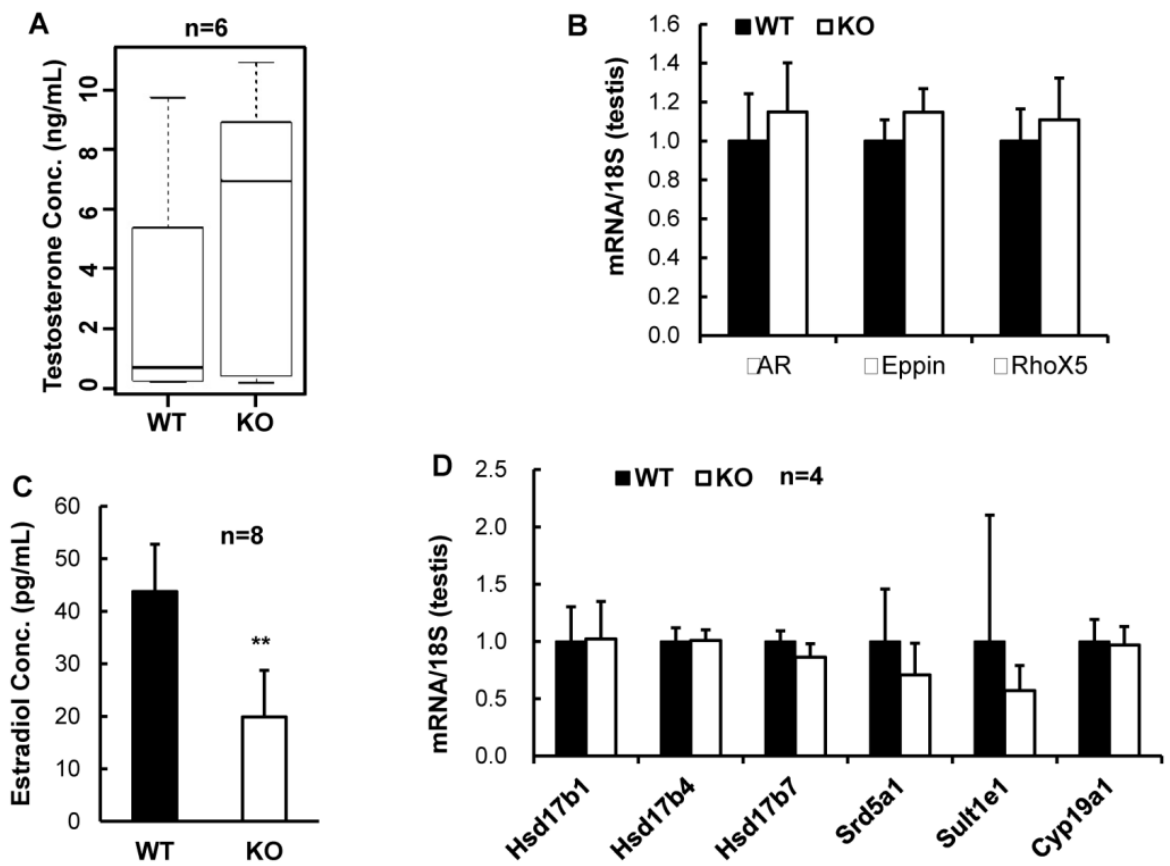

Figure 5. Analysis of steroid hormones and their relevant gene expression. A. The Box-Whisker plot of testosterone concentrations in the serum samples of $10-12$ week old male WT $(n=6)$ and Kdm3bKO $(K O)$ mice $(n=6)$. B. Quantitative RT-PCR analysis of the mRNA expression levels of AR and its target genes including Eppin and RhoX5 in the WT $(n=4)$ and Kdm3bKO mouse testes $(n=4)$. The data were normalized to the endogenous levels of $18 \mathrm{~S}$ RNA. C. 17 $\beta$-estrodial concentrations in the serum samples of 12 -week old male WT $(n=8)$ and Kdm3bKO mice $(n=8)$. **, $p<0.01$ by Student's $t$ test. D. The mRNA expression levels of estrogen biosynthesis and metabolism-related genes in the testes of WT $(n=4)$ and Kdm3bKO $(n=4)$ mice. The relative expression levels of these mRNAs were normalized to the endogenous 18 S RNA levels.
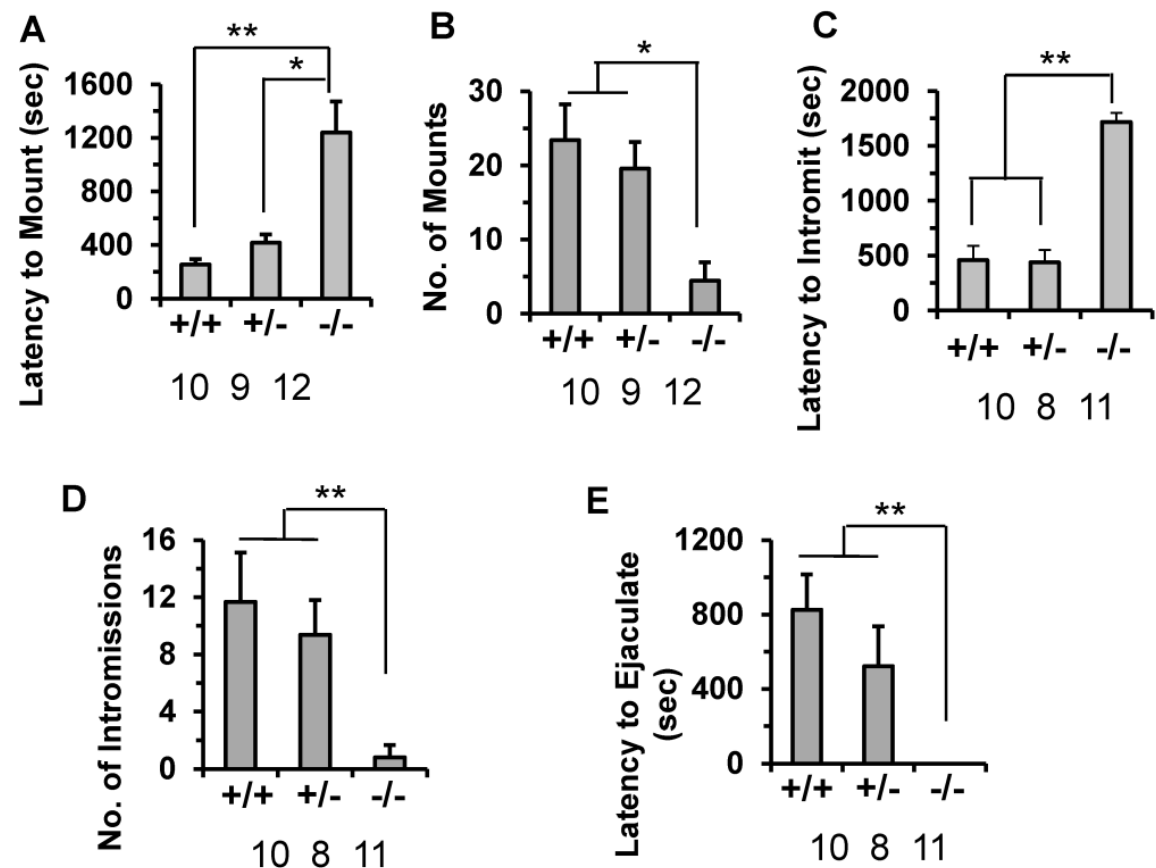

Figure 6. Knockout of $K d \mathbf{m} 3 \mathbf{b}$ in male mice altered their sexual behaviors. A \& B. The average latency time that $8-w e e k-o l d ~ W T(+/+, \mathrm{n}=10)$, heterozygous $(+/-, \mathrm{n}$ $=9)$ and $\mathrm{Kdm} 3 \mathrm{bKO}(-/-, \mathrm{n}=12)$ male mice took to mount the stimulus females, and the total number of mounts that these male mice carried out during the testing period. $*$ \& **, $\mathrm{p}<0.05$ and $\mathrm{p}<0.01$ by One-Way ANOVA. C \& D. The average intromission latency time that 8 -week-old WT $(n=10)$, heterozygous $(n=8)$ and Kdm3bKO $(n=11)$ male mice achieved their first intromission after the stimulus females were introduced, and the number of successful intromissions that these mice carried out during the testing period. **, $\mathrm{p}<0.01$ by One-Way ANOVA. E. The average latency time that 8 -week-old WT $(n=10)$, heterozygous $(n=8)$ and Kdm3bKO $(n=11)$ male mice took to ejaculate after the stimulus females were introduced. **, $\mathrm{p}<0.01$ by One-Way ANOVA. 
A

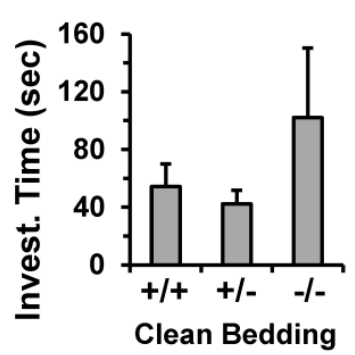

10912

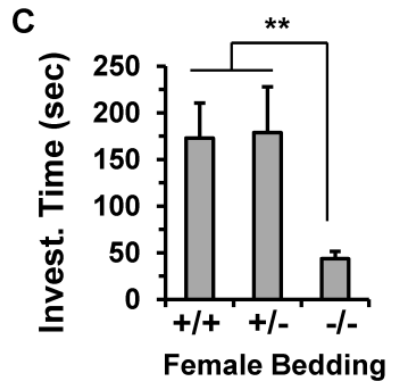

10912
B

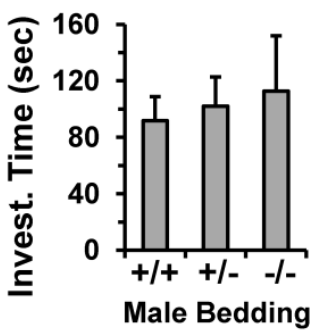

10912

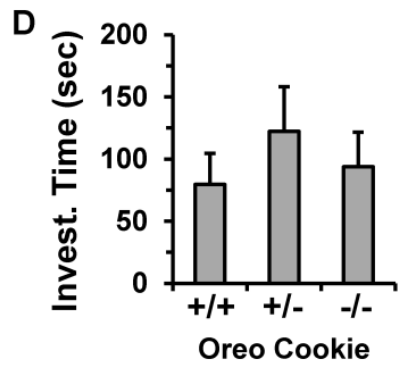

10912

Figure 7. Knockout of $K d m 3 b$ impaired the motivational components of the male sexual behaviors. The average time that $W T(+/+), n=10)$, heterozygous $(+/-$, $\mathrm{n}=9)$ and $\mathrm{Kdm} 3 \mathrm{bKO}(-/-, \mathrm{n}=12)$ male mice spent on investigating the clean bedding materials (Panel $A)$, the bedding materials with male odor (Panels $B)$, the bedding materials with female odor (Panel C), and the Oreo cookie (Panel D). **, $p<0.01$ by One-Way ANOVA.

\section{Discussion}

Although the enzyme activity and specificity of Kdm3b, a histone H3K9 demethylase, have been well studied, its physiological functions are largely unknown. To learn the genetic and physiological functions of the $K d m 3 b$ gene, we have recently generated $\mathrm{Kdm} 3 \mathrm{bKO}$ mice [16]. Through characterizing the growth phenotype and the female reproductive function, we found that $\mathrm{Kdm} 3 \mathrm{~b}$ is required for normal somatic growth and female reproductive function in mice [16]. In the present study, we further found that $\mathrm{Kdm} 3 \mathrm{~b}$ protein is expressed in Leydig cells, Sertoli cells, spermatocytes at different stages, and epithelial cells of the epididymis, seminal vesicle and prostate. We also found that knockout of $K d m 3 b$ in male mice decreased their sperm number and motility and compromised their both consummatory and motivational sexual behaviors. These abnormalities were tightly associated with markedly reduced male reproductive capability. Together, these findings demonstrate that $\mathrm{Kdm} 3 \mathrm{~b}$ is required for normal spermatogenesis, sperm maturation, and male reproductive function.

In this study, we showed that Kdm3bKO male mice produced $44 \%$ and $6 \%$ fewer numbers of total and mobile sperm, respectively, when compared with WT male mice. This phenotype may be explained by the knockout of $K d m 3 b$ expression and function in both spermatocytes and Sertoli cells. On the other hand, the numbers of total and mobile sperm gener- ated in the testes have big variations even among individual WT male mice and the partial decreases in these numbers within certain ranges may not be sufficient to significantly decrease the male reproductive function. For $\mathrm{Kdm} 3 \mathrm{bKO}$ male mice, this notion seems supported by the analysis of breeding data. When pairing with WT females, the reduced pup number from the breeding pairs with $\mathrm{Kdm} 3 \mathrm{bKO}$ male mice was mainly attributed to the decreased number of litters, but not the number of pups per litter, suggesting that the impaired male reproductive function may not due to the decreased number of sperm ejaculated each time, but to the reduced mating or pregnant frequency.

The results observed from the sexual behavior assays may help to explain the causes responsible for the decreased mating or pregnant frequency of the breeding pairs with $\mathrm{Kdm} 3 \mathrm{bKO}$ male and WT female mice. Our results demonstrate that $\mathrm{Kdm} 3 \mathrm{bKO}$ male mice exhibited increased latencies of mount, intromission and ejaculation after the stimulus females were introduced. These $\mathrm{Kdm} 3 \mathrm{bKO}$ male mice also exhibited decreased numbers of mounts and intromissions during the testing period. These observations clearly indicate that $\mathrm{Kdm} 3 \mathrm{~b}$ is required for maintaining the consummatory components of the male sexual behavior. Our results also demonstrate that $\mathrm{Kdm} 3 \mathrm{bKO}$ mice showed little interest in investigating the female-conditioned bedding material, indicating that $\mathrm{Kdm} 3 \mathrm{~b}$ is also required for the motivational sexual behavior. Taken together, the compro- 
mised male reproductive function of $\mathrm{Kdm} 3 \mathrm{bKO}$ mice could be a consequence of impaired motivational sexual behavior. Conceivably, the largely reduced sexual interest of $\mathrm{Kdm} 3 \mathrm{bKO}$ male mice in the stimulus females would reduce the performance of their consummatory sexual behavior, namely, the reduced frequencies of successful mating and female pregnancy.

Male sexual behaviors are regulated by sexual hormones including testosterone and estradiol in the brain [22]. An estrogen surge in the male brain at the neonatal stage is required for masculinizing and defeminizing. In adulthood, testosterone and estrogen are also required for activating and amplifying the male sexual behaviors. In the present study, we found that $17 \beta$-estradiol was markedly decreased in $\mathrm{Kdm} 3 \mathrm{bKO}$ male mice, although its responsible molecular mechanism was unclear and not due to any changes of estrogen synthetic or metabolic enzyme expression. This decreased estrogen level may be related to the compromised sexual behaviors of these male mice, since it has been shown that aromatization of testosterone into estrogen in the brain is important for masculinization of neurons that control male sexual behaviors [27].

Estrogen binds to and activates both estrogen receptor alpha and beta (ERa and ER $\beta$ ) to regulate their downstream genes. It has been shown that the activated ERa masculinizes, whereas ER $\beta$ defeminizes male mouse brain [22]. For transcriptional activation, both receptors recruit coregulator complexes containing epigenetic modification enzymes such as SRC-1, CBP and NcoR. These factors have been shown to express dimorphically in the male and female brains for modulating sexual behaviors [28]. As a histone modifier, $\mathrm{Kdm} 3 \mathrm{~b}$ contains a LXXLL (L, leucine; $X$, any amino acid residue) motif, which is known as a functional motif of steroid hormone receptor binding. Moreover, we detected abundant expression of $\mathrm{Kdm} 3 \mathrm{~b}$ in the brain by Western blot (data not shown). Therefore, one speculated mechanism for $\mathrm{Kdm} 3 \mathrm{~b}$ to regulate sexual behaviors might be that $\mathrm{Kdm} 3 \mathrm{~b}$ serves as a coactivator for ERa and/or ERb to mediate gene expression essential for male sexual behaviors. Another possibility is that the tissue-specific expression pattern of $\mathrm{Kdm} 3 \mathrm{~b}$ might be related to its role in regulation of male sexual behaviors. Although testis is the major source of circulating estrogen, brain, fat, uterus and other tissues also express the aromatase as well as estrogen-degradation enzymes according to the GenAtlas database. Thus, $\mathrm{Kdm} 3 \mathrm{~b}$ might regulate gene expression in these tissues to modulate estrogen production and male sexual behaviors. However, it is currently unknown how $\mathrm{Kdm} 3 \mathrm{~b}$ expresses and functions in the brain and other tissues to regulate the male sexual behaviors. It should warrant additional efforts and studies to answer these unresolved questions by investigators with appropriate expertise in neuroscience.

Kdm3a (JMJD1A, JHDM2A or TSGA) and $\mathrm{Kdm} 3 \mathrm{~b}$ (JMJD1B, JHDM2B or 5qNCA) are two of the three functionally related members in the KDM3 histone demethylase family. Both $\mathrm{Kdm} 3 \mathrm{a}$ and $\mathrm{Kdm} 3 \mathrm{~b}$ can interact with AR and demethylate H3K9me1/2 $[12,29,30]$. Our results showed that AR and several AR target genes were comparably expressed in WT and $\mathrm{Kdm} 3 \mathrm{bKO}$ mouse testes, suggesting that $\mathrm{Kdm} 3 \mathrm{~b}$ may not be essential for AR function in the mouse testis. In the germ cells, $\mathrm{Kdm} 3 \mathrm{a}$ and $\mathrm{Kdm} 3 \mathrm{~b}$ exhibit similar expression patterns and highly express in the pachytene cells. We have reported that knockout of $K d m 3 a$ caused severe defects in spermatogenesis and complete male infertility [4], suggesting that $K d m 3 b$ is unable to compensate the lost function of $\mathrm{Kdm} 3 \mathrm{a}$ in the germ cells of $K d m 3 a$ knockout mice during spermatogenesis. On the other hand, knockout of $K d m 3 b$ only partially affected spermatogenesis by reducing sperm number and motility, suggesting that $\mathrm{Kdm} 3 \mathrm{~b}$ may play a less important role than $\mathrm{kdm} 3 \mathrm{a}$ in supporting spermatocyte meiosis and differentiation. This notion is further supported by the different global changes of H3K9 methylation observed in the spermatocytes of $K d m 3 a$ and $K d m 3 b$ knockout male mice. In the testes of $K d m 3 a$ knockout mice, Western blot and immunohistochemistry detected significantly increased $\mathrm{H} 3 \mathrm{~K} 9 \mathrm{me} 1$ and $\mathrm{H} 3 \mathrm{~K} 9 \mathrm{me} 2$ in the pachytene spermatocytes and early elongating spermatids [4]. In contrast, we did not observe obvious changes of $\mathrm{H} 3 \mathrm{~K} 9 \mathrm{me} 1$ and $\mathrm{H} 3 \mathrm{~K} 9 \mathrm{me} 2$ in the testes of $\mathrm{Kdm} 3 \mathrm{bKO}$ mice, although we could not rule out the possibility that $K d m 3 b$ knockout might change the levels of these H3 methylation codes at certain specific gene loci. Interestingly, the opposite functional relationship is true between $\mathrm{Kdm} 3 \mathrm{a}$ and $\mathrm{Kdm} 3 \mathrm{~b}$ in the female reproductive organs. The $K d m 3 a$ female knockout mice with a 129SvEv and C57BL/6 mixed strain background have nearly normal reproductive function, while the $K d m 3 b K O$ female mice have significantly elevated levels of H3K9me1, H3K9me2 and H3K9me3 in their ovaries and uteri and are completely infertile $[4,16]$. Collectively, these observations suggest that $\mathrm{Kdm} 3 \mathrm{~b}$ cooperates with its family members such as $\mathrm{Kdm} 3 \mathrm{a}$ to regulate multiple physiological functions in a tissue and cell type-specific manner. More specifically, Kdm3a plays a more important role than $\mathrm{Kdm} 3 \mathrm{~b}$ in the testis to regulate spermatogenesis, while $\mathrm{Kdm} 3 \mathrm{~b}$ plays a more important role than $\mathrm{Kdm} 3 \mathrm{a}$ in the ovary, uterus and brain to regulate ovulation, fertilization, implantation and embryo 
growth in females, as well as sexual behaviors in males.

\section{Acknowledgements}

We thank the BCM Genetically Engineered Mouse Core for microinjection of ES cells, and this core is partially supported by a cancer center grant (P30-CA125123) from National Institutes of Health (NIH). This study was supported by NIH grants CA193455, CA112403, DK059820 and HD062512, and Cancer Prevention and Research Institute of Texas grants RP120732-P5 and RP150197.

\section{Competing Interests}

The authors have declared that no competing interest exists.

\section{References}

1. Russell LD, Ettlin RA, SinhaHikim AP, et al. Histological and histopathological evaluation of the testis. Clearwater, Fl: Cache River Press; 1990.

2. De Gendt K, Verhoeven G. Tissue- and cell-specific functions of the androgen receptor revealed through conditional knockout models in mice. Mol Cell Endocrinol. 2012; 352: 13-25.

3. Chimento A, Sirianni R, Casaburi I, et al. Role of estrogen receptors and $g$ protein-coupled estrogen receptor in regulation of hypothalamus-pituitary-testis axis and spermatogenesis. Front Endocrinol (Lausanne). 2014; 5: 1

4. Liu Z, Zhou S, Liao L, et al. Jmjd1a demethylase-regulated histone modification is essential for cAMP-response element modulator-regulated gene expression and spermatogenesis. J Biol Chem. 2010; 285: 2758-70.

5. Rathke C, Baarends WM, Awe S, et al. Chromatin dynamics during spermiogenesis. Biochim Biophys Acta. 2014; 1839: 155-68.

6. Song N, Liu J, An S, et al. Immunohistochemical Analysis of Histone H3 Modifications in Germ Cells during Mouse Spermatogenesis. Acta Histochem Cytochem. 2011; 44: 183-90.

7. Dada R, Kumar M, Jesudasan $R$, et al. Epigenetics and its role in male infertility. J Assist Reprod Genet. 2012; 29: 213-23.

8. An J, Zhang X, Qin J, et al. The histone methyltransferase ESET is required for the survival of spermatogonial stem/progenitor cells in mice. Cell Death Dis. 2014; 5: e1196.

9. Kuroki S, Akiyoshi M, Tokura M, et al. JMJD1C, a JmjC domain-containing protein, is required for long-term maintenance of male germ cells in mice. Biol Reprod. 2013; 89: 93

10. Peters $\mathrm{AH}$, O'Carroll D, Scherthan $\mathrm{H}$, et al. Loss of the Suv39h histone methyltransferases impairs mammalian heterochromatin and genome stability. Cell. 2001; 107: 323-37.

11. Tachibana M, Nozaki M, Takeda $N$, et al. Functional dynamics of H3K9 methylation during meiotic prophase progression. EMBO J. 2007; 26: 3346-59.

12. Kim JY, Kim KB, Eom GH, et al. KDM3B is the H3K9 demethylase involved in transcriptional activation of $1 \mathrm{mo} 2$ in leukemia. Mol Cell Biol. 2012: 32: 2917-33.

13. $\mathrm{Hu} \mathrm{Z}$, Gomes I, Horrigan SK, et al. A novel nuclear protein, 5qNCA (LOC51780) is a candidate for the myeloid leukemia tumor suppressor gene on chromosome 5 band q31. Oncogene. 2001; 20: 6946-54

14. MacKinnon RN, Kannourakis G, Wall M, et al. A cryptic deletion in $5 \mathrm{q} 31.2$ provides further evidence for a minimally deleted region in myelodysplastic syndromes. Cancer Genet. 2011; 204: 187-94.

15. Paolicchi E, Crea F, Farrar WL, et al. Histone lysine demethylases in breast cancer. Crit Rev Oncol Hematol. 2013; 86: 97-103.

16. Liu Z, Chen X, Zhou S, et al. The histone H3K9 demethylase Kdm3b is required for somatic growth and female reproductive function. Int J Biol Sci. 2015; 11: 494-507.

17. Chen X, Qin L, Liu Z, et al. Knockout of SRC-1 and SRC-3 in Mice Decreases Cardiomyocyte Proliferation and Causes a Noncompaction Cardiomyopathy Phenotype. Int J Biol Sci. 2015; 11: 1056-72

18. Lee DK, Liu Y, Liao L, et al. The prostate basal cell (BC) heterogeneity and the p63-positive BC differentiation spectrum in mice. Int J Biol Sci. 2014; 10: 1007-17.

19. Mark M, Yoshida-Komiya $\mathrm{H}$, Gehin $\mathrm{M}$, et al. Partially redundant functions of SRC-1 and TIF2 in postnatal survival and male reproduction. Proc Natl Acad Sci U S A. 2004; 101: 4453-8.

20. Oyola MG, Zuloaga DG, Carbone D, et al. CYP7B1 Enzyme Deletion Impairs Reproductive Behaviors in Male Mice. Endocrinology. 2015; 156: 2150-61.

21. Schauwaers K, De Gendt K, Saunders PT, et al. Loss of androgen receptor binding to selective androgen response elements causes a reproductive phenotype in a knockin mouse model. Proc Natl Acad Sci U S A. 2007; 104: 4961-6.

22. Yang CF, Shah NM. Representing sex in the brain, one module at a time. Neuron. 2014; 82: 261-78.

23. Eddy EM, Washburn TF, Bunch DO, et al. Targeted disruption of the estrogen receptor gene in male mice causes alteration of spermatogenesis and infertility. Endocrinology. 1996; 137: 4796-805.

24. Ferlin A, Ganz F, Pengo M, et al. Association of testicular germ cell tumor with polymorphisms in estrogen receptor and steroid metabolism genes. Endocr Relat Cancer. 2010; 17: 17-25.

25. Shehu A, Albarracin C, Devi YS, et al. The stimulation of HSD17B7 expression by estradiol provides a powerful feed-forward mechanism for estradiol biosynthesis in breast cancer cells. Mol Endocrinol. 2011; 25: 754-66.

26. Ye L, Su ZJ, Ge RS. Inhibitors of testosterone biosynthetic and metabolic activation enzymes. Molecules. 2011; 16: 9983-10001.

27. Wu MV, Manoli DS, Fraser EJ, et al. Estrogen masculinizes neural pathways and sex-specific behaviors. Cell. 2009; 139: 61-72.

28. Gagnidze K, Weil ZM, Pfaff DW. Histone modifications proposed to regulate sexual differentiation of brain and behavior. Bioessays. 2010; 32: 932-9.

29. Lim S, Metzger E, Schule R, et al. Epigenetic regulation of cancer growth by histone demethylases. Int J Cancer. 2010; 127: 1991-8.

30. Brauchle M, Yao Z, Arora R, et al. Protein complex interactor analysis and differential activity of KDM3 subfamily members towards H3K9 methylation. PLoS One. 2013; 8: e60549. 